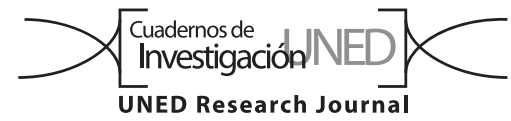

\title{
Manejo nutricional, sanitario, reproductivo y ambiental de 26 criaderos de perros (Canis lupus) en la Gran Área Metropolitana en Costa Rica
}

\author{
Mario Torres-Vargas' ${ }^{1} \&$ Rodolfo WingChing-Jones ${ }^{2}$ \\ 1. Universidad de Costa Rica, Escuela de Zootecnia, San José, Costa Rica; mtorres1388@gmail.com, (DD https://orcid.org/0000-0002-2347-161X \\ 2. Universidad de Costa Rica, Escuela de Zootecnia, Centro de Investigación en Nutrición Animal (CINA) San José, Costa Rica; \\ rodolfo.wingching@ucr.ac.cr, (iD https://orcid.org/0000-0002-8009-2210
}

Recibido 03-V-2019 • Corregido 29-VII-2019 • Aceptado 01-IX-2019

DOI: https://doi.org/10.22458/urj.v11i3.2719

\begin{abstract}
Nutritional, sanitary, reproductive and environmental management of 26 dog breeding kennels (Canis lupus) in the Great Metropolitan Area in Costa Rica". Introduction: Dog breeding kennels are establishments dedicated to the reproduction, breeding and trade of one or more breeds, either occasionally or permanently. Objective: We intend to analyze the activities that take place in registered kennels to understand how breeding kennels work in the Great Metropolitan Area (GAM, for its acronym in Spanish) in Costa Rica. Methods: In 2017 and 2018 we contacted the National Service of Animal Health (SENASA, for its acronym in Spanish) and three private associations related to breeding and trade activities in the country. Using survey tools, we collected the information related to animal management, feeding, hygiene, reproduction, health, facilities, and waste disposal. Results: We identified a total of 111 dog breeding production systems in the national territory, according to the lists of four entities, from which only $26,13 \%$ are registered in the regulating entity, SENASA. From the 26 kennels visited in the GAM, 23 different dog breeds are bred and managed, and the sale prices for these animals fluctuate between $\$ 500$ and $\$ 3500$. In the case of nutrition management, we identified balanced diets and the additional use of nutritional supplements to improve nutrient intake, but these could cause an excess of the nutritional requirements. As for reproduction, ultrasounds and progesterone tests are used to determine the best moment for the mounting. To manage waste and cleanliness, the management is similar between systems, where producers take charge or receive the support of an external party. Conclusions: Every dog breeding kennel system in the Great Metropolitan Area in Costa Rica has its own management strategies, which depend on the experience and technical support processes received by those in charge of the process.
\end{abstract}

Key words: breeding, reproduction, feeding, facilities, animal handle.
RESUMEN: Introducción: Los criaderos de perros son establecimientos dedicados a la reproducción, cría y venta de una o más razas, de forma ocasional o permanente. Objetivo: Pretendemos analizar las actividades que se realizan en criaderos registrados, para entender el funcionamiento de los criaderos en la Gran Área Metropolitana en Costa Rica. Métodos: Procedimos a contactar al Servicio Nacional de Salud Animal (SENASA) y tres asociaciones privadas relacionadas con la actividad de la cría y venta de caninos en el país, durante los años 2017 y 2018. Mediante un instrumento tipo encuesta, recolectamos la información relacionada al manejo de los animales, alimentación, higiene, reproducción, sanidad, instalaciones y manejo de desechos. Resultados: Determinamos un total de 111 sistemas de producción dedicados a la crianza de perros en todo el territorio nacional según las listas de los cuatro entes visitados, de los cuales solo el $26,13 \%$ se encuentran registrados ante el ente regulador, SENASA. De los 26 criaderos vistados en el GAM, se crían y manejan 23 razas y los precios de venta de estos animales, fluctúan entre $\$ 500$ hasta un máximo de $\$ 3$ 500. En el caso del manejo alimenticio encontramos el uso de suplementos alimenticios adicionales al uso del alimento balanceado, para mejorar el aporte de nutrimentos en la dieta, los cuales podrían provocar un aporte que sobrepase los requerimientos nutricionales. En la reproducción, sobresale el uso de ultrasonidos y exámenes de progesterona para determinar el momento de la monta. Para el manejo de desechos y limpieza de caniles, el manejo presenta similitud entre sistemas, donde los productores se hacen cargo o reciben apoyo de una persona externa. Conclusión: Los criaderos de perros en la gran área metropolitana en Costa Rica presentan estrategias de manejo propias de cada sistema, las cuales se asocian a la experiencia y procesos de apoyo técnico que reciben o recibieron los encargados de los sistemas.

Palabras clave: Crianza, reproducción, alimentación, instalaciones, manejo de animales.
Los perros son la principal mascota en los hogares de latinoamerica. En un estudio realizado en 22 países del mundo, el $56 \%$ de los entrevistados indicaron convivir con una mascota, donde esta fue un perro (33\%) (GfK, 2016). De igual manera a los resultados expuestos, en
Costa Rica en el año 2012, se indicó que el $85 \%$ de las personas que responde tener un animal de compañía, afirmaron que era un perro (Camacho, 2012), situación que disminuyó para el año 2016, a 50,5\% (WAP, 2016). Este comportamiento en la tenencia de perros, provocó 
un crecimiento en el mercado de alimentos balanceados para mascotas, donde el costarricense en promedio invierte en la alimentación de su mascota \9 425 mensuales (\$17,4 dólares americanos).

La forma en que el perro llega a los hogares se resume en procesos de adopción, como regalo o la compra en un criadero. En el caso de los criaderos, son establecimientos dedicados a la reproducción, cría y venta de una o más razas, de forma ocasional o permanente, donde el criador es el responsable de que se cumplan todas las medidas dictadas en el Reglamento para la Reproducción y Tenencia Responsable de Animales de Compañía en Costa Rica, según lo dicta el Servicio Nacional de Salud Animal (SENASA) (2004). Además de este reglamento, se cita la Ley 7451 del 16 de Noviembre de 1994: Ley de Bienestar de los Animales, la Ley 8495 del 6 de Abril del 2006: Ley General del Servicio Nacional de Salud Animal y la Ley 9245 del 7 de Mayo del 2014: Ley contra las peleas de caninos.

En ocasiones, la venta de estos animales se realiza en lugares que no cumplen con los reglamentos o leyes existentes, y se limita a ser una actividad económica y sin que se tome en cuenta la calidad genética y el bienestar animal, lo cual podría provocar problemas nutricionales, defectos genéticos o agresividad en el animal. En Costa Rica, el Servicio Nacional de Salud Animal (SENASA) del Ministerio de Agricultura y Ganadería (MAG), decomisó 390 perros que se encontraban en situación de crueldad, abandono y utilizados para peleas en el Área Metropolitana en el 2014 (Arce, 2015).Para los años 2013 y 2014 se registraron 1000 y 1200 denuncias, mientras que en el año 2015, se atendieron 1867 , relacionadas a perros bravos $(41,51 \%)$, mordeduras de perros $(17,51 \%)$, maltratos o agresión (12,48\%), tenencia irresponsable $(23,09 \%)$, criadros ilegales (2,09\%), acumulación de perros $(3,05 \%)$ y $0,27 \%$ sin descripción de la denuncia (Gutierrez, 2016).

Los criaderos son los lugares de preferencia por los costarricenses para la adquisición de los perros por lo cual conocer estos sitios, como los centros de adopción y los hogares en los que se van a criar (Konok et al., 2015), permite evaluar el manejo alimenticio, sanitario, reproductivo, instalaciones y las prácticas de bienestar animal que se implementan a estos animales. Por tal motivo, en este estudio pretendemos analizar las actividades que se realizan en criaderos registrados, al evaluar las prácticas de manejo de animales en las etapas de cachorro hasta animal adulto, alimentación, limpieza y sanidad de instalaciones como de animales, dimensiones de las instalaciones, manejo de desechos, entre otros factores que permitan generar el conocimiento para entender cómo es que funcionan los criaderos de perros registrados en la Gran Área Metropolitana en Costa Rica.

\section{MATERIALES Y METODOLOGÍA}

Ubicación de los sistemas de producción: Para ubicar los criaderos de perros inscritos que se encuentran en funcionamiento en la Gran Área Metropolitana (GAM) en Costa Rica, durante los años 2017 y 2018, se procedió a contactar al Servicio Nacional de Salud Animal (SENASA), la Asociación Canófila Costarricense (ACC), la Federación Canina Costarricense (FCC) y la Asociación del Ovejero Alemán de Costa Rica (ASOVAL), institución del estado y organizaciones privadas relacionadas con la actividad de la cría y venta de caninos en el país, con la intención de obtener la lista de criaderos registrados en cada una de ellas.

Después de obtener y analizar las listas de criaderos asociados a estas instancias, se procedió a unificarlas cuatro bases de datos, descartando las unidades que se encontraban repetidas en una o varias listas. La información resultante se organizó por medio de la ubicación geográfica del establecimiento en Costa Rica, descartando aquellos que se encontraron fuera de la GAM, la cuál esta delimitada al Norte con las estribaciones de la Cordillera Volcánica Central en Heredia y Alajuela; al Sur las montañas de Aserrí (Provincia de San José); al Este el cantón de Paraíso (Provincia de Cartago) y al Oeste con Atenas (Provincia de Alajuela) (Vargas, 2006).

Se confeccionó un instrumento de recolección tipo encuesta, por medio del cual se obtuvo información zootécnica de interés relacionada a los criaderos como la raza de animal que crían, precios de venta, nivel educativo de los cuidadores, el uso de registros, cantidad de alimento y tiempos de alimentación de los animales, manejo del alimento, dimensiones y limpieza de las instalaciones, manejo sanitario, bioseguridad, manejo reproductivo y manejo de desechos.

Análisis de la información: La información recopilada se tabuló en hojas de cálculo, donde se mantuvo concordancia del criadero analizado y las respuestas obtenidas. Se tabularon 26 registros, en donde se consideraron variables (alimentación, sanidad y caniles) e indicadores de cada sistema (tiempos de alimentación, cantidad ofrecida), así como también la actividad de cada lugar (si es solo criadero, criadero y hospedaje, criadero hospedaje y entrenamiento). Los resultados obtenidos, se presentan en forma descriptiva como porcentaje, el cual se obtuvo de la sumatoria de criaderos que responden de forma 
similar para una variable entre el total de criaderos vistados (26), multiplicado por 100.

Ética, conflicto de intereses y declaración de financiamiento: Los autores declaran haber cumplido con todos los requisitos éticos y legales pertinentes, tanto durante el estudio como en el manuscrito; que no hay conflictos de interés de ningún tipo, y que todas las fuentes financieras se detallan plena y claramente en la sección de agradecimientos. Asimismo, están de acuerdo con la versión editada final del documento. El respectivo documento legal firmado se encuentra en los archivos de la revista.

\section{RESULTADOS}

Se identificó un total de 111 sistemas de producción dedicados a la crianza de perros en todo el territorio nacional según las listas de las cuatro organizaciones visitadas, de los cuales solo el $26,13 \%$ se encuentran registrados ante el ente regulador, SENASA. En el caso de la ACC solo el 7,84\% de sus criaderos están inscritos. Para la FCC, su único criadero, se encuentra registado y de los 36 criaderos que componen la ASOVAL ninguno aparece en la lista suministrada por el SENASA.

De los 26 criaderos vistados en el GAM, 19 crían y manejan una raza, cuatro criaderos dos razas y tres de ellos más de dos razas. Los precios de venta de estos animales, fluctúan entre \$500 hasta un máximo de \$3 500 según la especie (Cuadro 1). El 76,92\% de los casos, la edad de entrega de los cachorros es entre dos y dos meses y medio, y el monto a cancelar por la venta de cachorro es el día de la entrega (54\%), existiendo otros métodos de pago, según el servicio brindado, como la venta del animal, entrenamiento, hospedaje o la combinación de servicios. En cuanto a hospedaje o entrenamiento, el $80 \%$ de los criaderos toman datos de los dueños y solicita historial de vacunas (80\%).

En aspectos administrativos, se determinaron diferencias entre establecimientos en relación al manejo de la información del cliente, el nivel acádemico del personal, los servicios que prestan y el costo de estos servicios (Fig. 1 en Apéndice Digital). La mayoría de los sistemas productivos, se dedican a la crianza, el nivel académico se limita a educación media por parte de los cuidadores y nivel profesional de las personas que los aseroran o les brindan apoyo técnico (73\%). De igual manera, las medidas de bioseguridad implementadas en los sistemas productivos, son pocas (cuarentena, control de plagas y pediluvios) y que el manejo de los desechos orgánicos producidos es por los responsables del sistema (Fig. 1 en Apéndice Digital).

En cuanto a las instalaciones el $77 \%$ de los corrales o caniles, en un mismo criadero, presentaron un tamaño similar y fue utilizado para el cuido y manejo de un animal. En cuanto a la sanidad y limpieza de los caniles, el método fue el mismo en todos los criaderos, el cual consistió en la recolección de desechos sólidos, enjaugue conagua, limpieza con jabón y desinfectante; y posterior lavado con agua, y un periodo de espera, para que se seque el canil e ingresar al animal. En cuanto al manejo de

CUADRO 1

Razas presentes en los 26 criaderos encuestados y el ámbito de precio de cada animal comercializado en su etapa fisiológica de animal como cachorro

\begin{tabular}{llll} 
& \multicolumn{2}{c}{ Razas // Ámbito de Precio* } \\
Pastor Alemán & $\$ 500-\$ 1000$ & Dobermann & $\$ 600-\$ 2000$ \\
Labrador Retriever & $\$ 600-\$ 1000$ & Golden Retriever & $\$ 600-\$ 1000$ \\
Terranova & $\$ 2500$ & Bernés de la Montaña & $\$ 1500$ \\
American Bully & $\$ 1400-\$ 3500$ & Shar Pei & $\$ 600-\$ 1500$ \\
Rottweiler & $\$ 400-\$ 1500$ & Fila Brasileño & $\$ 1000-\$ 1200$ \\
Rhodesian Ridgeback & $\$ 1000-\$ 1500$ & Boxer & $\$ 500-\$ 1000$ \\
Border Collie & $\$ 1000-\$ 2000$ & Beagle & $\$ 500-\$ 2000$ \\
Cocker Inglés & $\$ 500-\$ 750$ & Pug & $\$ 1000-\$ 2000$ \\
Bulldog Inglés & $\$ 1200-\$ 1500$ & Boston Terrier & $\$ 250-\$ 1500$ \\
Dachshund Pelo Largo & $\$ 1400-\$ 2500$ & Pomeranian & $\$ 800-\$ 3000$ \\
BichónMaltés & $\$ 600-\$ 1000$ & Yorkshire Terrier & $\$ 600-\$ 800$ \\
Chihuahua & $\$ 600-\$ 700$ & &
\end{tabular}

*\$1 dólar americano $=\$ 600$ colones (Banco Central de Costa Rica, octubre 2018) 
las parideras el proceso es similar, solo que varía según el nivel de limpieza o la frecuencia. La desinfección de los caniles se realiza con cloro en $42 \%$ de los casos, ya sea solo o en conjunto con otros productos una vez a la semana. En cuanto al lavado de las bandejas de alimentación, en el $88 \%$ de los criaderos las lavan después de cada comida.

Se rescata de los resultados, la preocupación de los responsables por estar preparados ante una emergencia, donde el $100 \%$ cuenta con un botiquín, el cual diferenciaban entre botiquín completo, aquel que presenta paños, gaza, guantes, yodo, alcohol, algodón, materiales que son de utilidad para recibir un parto $y / 0$ afrontar una herida que no requiera sutura; y el botiquín básico que presenta gaza y/o algodón, alcohol y yodo. Por otro lado, se encontrarón diferentes progamas de vacunación contra parvovirus, distemper y adenovirus, en cachorros antes de los tres meses, el cual varió según el médico veterinario que visitaba el sistema de producción.

En la alimentación de los cachorros y animales reproductores se encontraron diferentes sistemas de manejo, en los cachorros, se da un consumo libre de los alimentos (ad libitum). Se encontraron casos en los que se ofrece alimento hidratado más carne molida, alimentación de forma individual, mezcla de carne y arroz; y mezcla de molida de hígado, corazón, riñones y testículos de res más medicamentos contra la artritis para evitar daños en huesos y evitar el uso de alimento balanceado. En el caso de animales para exposición, se les ofrece mayor cantidad de alimento y mezclado con carne de res o con alimento de cachorro. En otros casos, se les ofrece carne de res adicional tres veces a la semana, mezcla de arroz con carne de res más zanahoria, ayote, huevo duro y naranja después de la comida y huesos grandes como fémures de res para el cuidado de los dientes una vez por semana. En el caso de hembras gestantes adicional al alimento, se ofrecen suplementos de avena y miel, alimento de cachorro, leche de cabra, leche delactosada en la primera semana preparto. En hembras lactantes se ofrece avena, miel de aveja y leche de cabra, carne de res adicional. En la mayoría de los casos, la cantidad de suplementos está relacionada al tamaño de la camada y el peso del animal.

En la parte reproductiva, el manejo de los reemplazos, el recambio de hembras y machos; y la cantidad de animales varía dependiendo del criadero y la raza (Fig. 1 en Apéndice Digital). En el caso de la monta, se da una preferencia por la monta natural, donde la fecha y la frecuencia, se determina por la actitud del macho reproductor y experiencia del criador, y en otros casos, depende de los exámenes de progesterona, debido a que permiten determinar con mayor certeza el momento de la ovulación.
En el caso de que un animal no siga en el ciclo productivo del criadero, estos se quedan con el dueño (50\%) o reubicados en hogares de personas allegadas a los dueños. Estos son reemplazados por animales criados en el mismo criadero o obtenidos de otros establecimientos, bajo criterios de selección desarrollados por los responsables de cada unidad.

\section{DISCUSIÓN}

La situación encontrada en esta investigación con relación al bajo porcentaje de criaderos inscritos, genera preocupación por la falta de control por parte del SENASA (Ley 8495. Ley General del Servicio Nacional de Salud. Artículo 56) y de las asociaciones respectivas al momento en que se realizó este trabajo. Con los resultados obtenidos en este trabajo, se logró describir y caraterizar el manejo zootécnico de estos sistemas al integrar los componentes de instalaciones, nutricionales, sanitarios, genéticos, administrativos y ambientales. Se observó que el $73 \%$ de los entrevistados reciben asesoramiento por profesionales en el campo nutricional, sanitario y de manejo de esta especie.

La reproducción de los perros es una actividad que se diversifica y no solo se limita a la cría de animales, además se ofrecen entrenamientos y hospedaje, combinación que permite captar ingresos entre $\$ 300$ y $\$ 3500$, según los servicios brindados. Esta situación se encuentra limitada por el número de compradores que entienden la importancia de tener un perro entrenado (Gómez, Atehortua \& Orozco, 2007), el número de cachorros que presenta una camada, la sobrevivencia de la misma, la oferta y demanda según la raza y la necesidad del dueño de que el perro optimice sus características físicas, psíquicas y técnico-tácticas (Pellegrino, et al.; 2014).

Se debe prestar atención a la presencia de 23 razas que se comercializan en el país, desde razas grandes, medianas y pequeñas (Case, Carey \& Hirakawa, 1997), siendo la raza pastor alemán, la que más se cría en el país. Es de esperar, debido a que por el proceso de domesticación que sufrieron los perros, es la especie que presenta una mayor selección artificial atraves del tiempo, que resulto en variedad de morfologías, genes y adaptación de ambientes (Svartberg, 2006). Sumado a los diferentes papeles o actividades que esta mascota cumpliría como animal de compañía (Cekavicius \& Pajarskaite, 2012).

En cuanto a las características de los caniles, el $61,5 \%$ de los criaderos visitados cuentan con dimensiones adecuadas, en donde la regla general fue de 1,5 $\mathrm{m}$ por 4 m. Según la RSPCA (Real Sociedad para la Prevención de la Crueldad a los Animales o Royal Society for the 
Prevention of Cruelty to Animals por sus siglas en Inglés) (2009) en jaulas individuales, se debe garantizar un mínimo de $2 \mathrm{~m}^{2}$ de alojamiento que lo proteja del viento y la lluvia, un espacio mínimo para ejercitarse de 2,5 a $3,5 \mathrm{~m}^{2}$, una cama elevada de la superficie y una malla de al menos $2 \mathrm{~m}$ de altura de malla electrosoldada e inclinada hacia adentro para evitar que los perros la escalen. De igual manera, para un canil para perros de raza grande, se recomienda ofrecer un total de $6 \mathrm{~m}^{2}$, donde una mitad sea cubierta y la otra de patio (Camps, 2015). Este autor menciona que el ancho de los caniles depende de su tamaño y pueden variar de $1,5 \mathrm{~m}$ de ancho por $2 \mathrm{~m}$ de profundidad hasta 2,5m de ancho por 1,2m de profundidad, en donde la regla general es que la superficie total sea de $1,5 \mathrm{~m}$ de ancho por $4 \mathrm{~m}$ de profundidad.

En el caso de la temperatura ambiental se recomienda de forma general una temperatura ambiental mínima de $10^{\circ} \mathrm{C}$ y no más de $26^{\circ} \mathrm{C}$. En la etapa comprendida desde el nacimiento al destete se requiere que la estructura permita mantener un ámbito entre 20 y $25^{\circ} \mathrm{C}$; mientras que, de 30 a $35^{\circ} \mathrm{C}$ durante la primera semana de nacidos. En adultos, la temperatura óptima en el interior del canil debería estar entre 15 y $25^{\circ} \mathrm{C}$ (Camps, 2015).

En relación al proceso de limpieza de los caniles, se debe limpiar las jaulas de animales jóvenes (cachorros) hasta animales adultos; por último, animales heridos o enfermos; sin importar las actividades que se realicen en el sistema (Criadero y/o hospedaje). Como se describió en los resultados, se debe limpiar la jaula de cualquier desperdicio sólido, como heces o pelaje y se debe enjuagar con agua caliente, en los sistemas visitados no se cumple el uso de agua caliente, pero es sustituido por un efecto físico provocado por el cepillo con el detergente. Posterior a esta primera fase de limpieza, se prepara la disolución desinfectante, según recomendaciones del fabricante y se aplica en todas las superficies, restregando toda la jaula (delante y detrás), en el techo y el piso y en las bisagras de las puertas; se debe secar con ayuda de un escurridor y ventilación y posterior a esto volver a introducir al animal a su jaula (Newbury et al., 2010).

En términos del manejo sanitario de los perros, los sistemas visitados presentan sus programas de vacunación y desparacitación, los cuales son importantes para evitar afección de parásitos y/o enfermedad que pueda afectar al animal (Nazario, 2015), sin dejar de lado, una adecuada alimentación, la cual es vital para una buena salud (Drocco, 2013) y el establecimiento y cumplimiento de medidas de bioseguridad.

Es importante recordar que la bioseguridad es la primera defensa que los criaderos pueden implementar para proteger sus animales de agentes microbiales provenientes del exterior que afecten la salud y el rendimiento de los animales, como también, la protección a lo interno entre las diferentes etapas fisiológicas presentes en el sistema (Dendoncker et al., 2018). Por ejemplo, en Bélgica, se encuentró que de 42 criaderos pequeños (< 10 hembras) el 17,1\% realiza un control de plagas, mientras que, de siete criaderos considerados grandes ( $>10$ hembras), el 87,7\% realiza este control. La diferencia de los resultados, se asocia a la importancia que le den a la actividad y al reconocimiento legal que se otorga a estos sistemas (Dendoncker et al., 2018). En el caso de los resultados obtenidos en esta investigación, se presentan oportunidades de mejora, en el uso de pediluvios, control de roedores y la implementación de periodos de cuarentena, por parte de los encargados de los criaderos de perros, para garantizar un ambiente que favorezca el desarrollo y cuido de los animales.

Otro aspecto a considerar en el manejo de los animales en los criaderos es la alimentación, factor importante durante el ciclo de vida de los perros y que debe promover una condición corporal acorde a la raza y edad; y reducir el riesgo de sufrir patologías nutricionales como la obesidad. Los resultados obtenidos en este trabajo, demuestran que según la apreciación del encargado o dueño, se ofrece una cantidad de alimento balanceado, más la adición de diferentes fuentes de alimento, con la intención de aumentar el aporte de nutrimentos y alcanzar una mayor tasa de crecimiento, práctica de alimentación que podría provocar desbalances nutricionales en los animales. Es importante tener claro, que la nutrición de los caninos se puede ver afectada por factores específicos del animal (edad, estado fisiológico, actividad), de la dieta (desequilibrio de nutrientes, deterioro, contaminación, adulteración), del manejo de la alimentación (frecuencia de alimentación, método de alimentación) y los factores ambientales (espacio, calidad del entorno) (Balwin et al., 2010). Según lo anterior, la alimentación suministrada al animal debe cumplir los requerimientos diarios de energía y proteína. En el caso de la determinación de los requerimientos, el uso de modelos mátematicos, permiten estimar las necesidades de energía de un perro. Por ejemplo, el cálculo de la energía metabolizable (EM), energía retenida por el organismo, es calculada usando la fórmula $\left(\mathrm{K} \times \mathrm{W}^{0,67}\right)$, donde $W$, es el peso del animal en kilogramos y $K$ es una constante de actividad, la cual puede tomar valores de 132 para un animal inactivo, 145 para un animal activo, 200 para un animal muy activo y 300 para animales de alto rendimiento (Case et al., 1997). En el caso de la proteína, nutrimento importante para el crecimiento por su aporte de aminoácidos, se considera un mínimo de 56,3g por cada $1000 \mathrm{kcal}$ de EM, para etapas de crecimiento y reproducción. Mientras 
que, para el mantenimiento de animales adultos, se estima un mínimo de 45g/1000 Kcal EM (AAFCO, 2014).

Por último, en aspectos reproductivos, las técnicas de asistencia reproductivas se han desarrollado con la intención de mejorar y controlar la reproducción de los animales domésticos, el uso de inseminación artificial, fertilización in vitro, producción de embriones, transferencia de embriones y crioconservación de gametos (Luvoni, 2000). En el caso de los criaderos vistados, en Costa Rica solo se implementa la verficiación de la preñez (Paz, 2015) y determinación del número de fetos que se están gestando. Situación que permite un desarrollo en el campo del manejo reproductivo de los animales en los criaderos de perros.

Limitaciones del estudio: El presente trabajo se limitó a la aceptación del responsable del criadero a participar. De los 111 criaderos presentes, 24(21,62\%) se encuentra fuera de la Gran Área Metropolitana y 61 (54,95\%) de los criaderos decidieron no partipar, aduciendo, no calificar para el estudio (15,31\%), no permiten el ingreso a terceros $(2,70 \%)$, no se dedican a la actividad $(6,31 \%)$ o no contestaron la solicitud (28,82\%).

\section{AGRADECIMIENTOS}

Los autores agradecen a lliana Céspedes (SENASA), a la Asociación Canófila Costarricense y sus asociados, a la Asociación Club Ovejero Alemán de Costa Rica y sus asociados; y a los criadores que se visitaron durante la realización de este proyecto por su ayuda, su sinceridad y su interés en el desarrollo de esteproyecto.

\section{REFERENCIAS}

Arce, R. (2015). Costa Rica: SENASA rescató cerca de 400 perros en 2014. Humane Society International/Latinoamérica. Press Releases. Recuperado de http://hsi-old.pub30. convio.net/spanish/news/press_releases/2015/01/costa-rica-rescate-perros-2014-012114.html

Association of American Feed Control Officials (AAFCO). (2014). AAFCO Methods for Substantiating Nutritional Adequacy of Dog and Cat Foods. Proposed Revisions Edited per Comments for 2014 Official Publication. Illinois, USA: AAFCO.

Balwin, K., Bartges, J., Buffington, T., Freeman, L., Grabow, M., Legred, J., \& Ostwald, D. (2010). Guías para la Evaluación Nutricional de perros y gatos de la Asociación Americana Hospitalaria de Animales (AAHA). Journal of the American Animal Hospital Association, 46(4), 285-297. DOI: $10.5326 / 0460285$
Camps, J. (2015). Planos y ambientes para caniles. Da Derecho Animal Web Center. Recuperado de http://www.derechoanimal.info/images/pdf/Caniles.pdf

Camacho, A. (2012, mayo). Ticos Chinean a sus Mascotas. Sección Inteligencia Financiera. Revista El Financiero. Recuperado de http://wvw.elfinancierocr.com/ef_archivo/2012/mayo/06/inteligencia2097516.html

Case, L., Carey, D., \& Hirakawa, D. (1997). Nutrición Canina y Felina. Manual para Profesionales. Madrid, España: Hartcourt Brace.

Cekavicius, T., Pajarskaite, M. (2012). Pets as status symbols (Tesis de maestría). Jonkpping University. Suecia. Recuperado de http://www.diva-portal.org/smash/ get/ diva2:531243/ FULLTEXT02

Dendoncker, P.A., Moons, C., Sarrazin, S., Diederich, C., Thiry, E., de Keuster, T., \& Dewulf, J. (2018). Biosecurity and management practices in different dog breeding systems have considerable margin for improvements. Veterinary Record, 183(12), 381. DOI: 10.1136/vr.104996

Drocco, A. (2013). Manejo Sanitario del Cachorro. Virbac. Laboratorios Santa Elena. Montevideo, Uruguay. Recuperado de http://www.santaelena. com.uy/ uc_126_1.html.

Gesellschaft Für Konsumforschung (GFK). (2016). La mayoría de quienes tienen mascota radican en América Latina, Rusia y los EE.UU. Estudios de Mercadeo. Insights. Comunicado de prensa. Núremberg. Alemania. Recuperado de http:// www.gfk.com/es-co/insights/press-release/la-mayoria-de-quienes-tienen-mascota-radican-en-america-latina-rusia-y-los-eeuu-2/

Gómez, L., Atehortua, C., \& Orozco, S. (2007). La influencia de las mascotas en la vida humana. Revista Colombiana de Ciencias Pecuarias, 20, 377-386.

Gutierrez, P. (2016). El maltrato y abuso a la vida animal (Proyecto de Licenciatura). San José, Costa Rica: Universidad de Costa Rica.

Konok, V., Kosztolányi, A., Rainer, W., Mutschler, B., Halsband, U., \& Miklósi, A. (2015). Influence of owner's attachment style and personality on their dog's (Canisfamiliaris) Separation-related disorder. PLOS ONE, 10(2), 17. DOI: 10.1371/journal.pone.0118375

Luvoni, G. (2000). Current progres son assisted reproduction in dogs and cats: in vitro embryo production. Reproduction Nutrition Development, 40, 505-512. DOI: 10.1051/ rnd:2000114

Nazario, L. (2015). Vacunación en perros y gatos. Guía para el bienestar animal. Universidad Técnica Nacional, Costa Rica. UTN Informa, 71, 5

Newbury, S., Blinn, M., Bushby, P., Barker, C., Dinnage, J., Griffin, B., Hurley, K., Isaza, N., Jones, W., Miller, L., O'Quin, J., Patronek, G., Smith-Blackmore, M. \& Spindel, M. (2010). Guidelines for standards of care in animal shelters. The Association of Shelter Veterinarians. Recuperado de: 
https://www.sheltervet.org/assets/docs/shelter-standards-oct2011-wforward.pdf

Paz, R. (2015). Uso de las determinaciones de progesterona en el manejo reproductivo de la perra. $1^{\circ}$ Congreso Internacional de Ciencias Veterinarias. FMVZ-UNAM. México. Recuperado de: http://congreso.fmvz. unam.mx/pdf/memorias/Ciencias\%20Veterinarias/ Progesterona\%20perra\%20Rafael\%20Paz\%20CCV.pdf

Pellegrino, F., Risso, A., Arias, D., Blanco, P. \& Corrada, Y. (2014). Optimización del rendimiento deportivo en caninos. Revista de Investigaciones Veterinarias del Perú, 25(4), 449-454.

Protección Animal Mundial (WAP). (2016). Estudio Nacional sobre tenencia de perros en Costa Rica 2016. San José, Costa Rica: WAP.
Royal Society for the Prevention of Cruelty to Animals (RSPCA). (2009). Guía para el diseño y manejo de un albergue para animales. United Kingdom: RSPCA International.

Senasa. (2004). Reglamento para la Reproducción y Tenencia Responsable de Animales de Compañía. № 31626S. La Gaceta N ${ }^{\circ}$ 26. Recuperado de http://www.hacienda.go.cr/centro/datos/Decreto/Decretos $\% 20$ 31620-G-Asueto-31626-S-Regl.\%20Animales-La\%20 Gaceta\%2026-6\%20FEB-2004.pdf

Svartberg, K. (2006). Breed-typical behaviour in dogs-Historical remnants or recent constructs?. Applied Animal Behaviour Science, 96, 293-313. DOI: 10.1016/j. applanim.2005.06.014

Vargas, G. (2006). Geografía de Costa Rica. San José, Costa Rica: EUNED. 